\title{
Human Activity Classification Using On-Body Miniaturized Antennas
}

\author{
Paritosh D. Peshwe ${ }^{1} \cdot$ Neha Y. Joshi $^{2}$ (D) . Ashwin G. Kothari ${ }^{2}$
}

Accepted: 4 January 2022 / Published online: 17 January 2022

(c) The Author(s), under exclusive licence to Springer Science+Business Media, LLC, part of Springer Nature 2022

\begin{abstract}
EM waves are extremely powerful when it comes to propagation of information during communication. There is no alternative to EM waves in such applications. However, the use of EM waves or antennas in general has not been explored fully as sensors for measuring the change in physical environment. This particular aspect has been exploited and the present work focuses on the application of antennas into the domain of classification. We propose accurate human activity classification (HAC) using on body miniaturized antennas. A simple patch antenna has been designed in order to be employed on human body for HAC. The antenna has been tested with respect to its Specific Absorption Ratio (SAR) values in order to make it body-mountable. The antenna has been fabricated and tested on human body while performing daily activities. The reflection co-efficient of antennas is a function of human motion activities and this principle is exploited to achieve the desired results. To explore different approaches, a miniaturized circularly polarized antenna is then designed and tested. The results of the two antennas are then compared. Dynamic Time Warping (DTW) algorithm has been used for the analysis of the Reflection co-efficient of the antenna. Excellent activity classification performance has been obtained using both the antennas, the miniaturized antenna giving better results.
\end{abstract}

Keywords Human activity classification (HAC) · On-body antennas

Neha Y. Joshi

nehayj100@gmail.com

Paritosh D. Peshwe

paritoshpeshwe@iiitn.ac.in

Ashwin G. Kothari

ashwinkothari@ece.vnit.ac.in

1 Electronics and Communication Department, Indian Institute of Information Technology, Nagpur, India

2 Electronics and Communication Department, Visvesvaraya National Institute of Technology, Nagpur, India 


\section{Introduction}

Human activity classification has become a prime field of research for the classification of actions performed by robots employed for human activities. Other areas such as physiotherapy, athlete training, surveillance etc. also employ HAC. Home-based treatment for physiotherapy can be dangerous if the exercise is not performed accurately. A Human Activity Classifier ( $\mathrm{HACl}$ ) can help the patient to know if the motion he has performed is accurate. Similarly HAC can help to know if some suspicious activity is being performed in public places like airports and hence can be used for surveillance. Currently, HAC has also gained a lot of importance in gesture based games. Gesture to speech converters can also employ HACls for easy operation.

The most common classifiers in literature are based on the principles of video processing. The video of the subject performing activity is captured and then analysed. Space time approach for HAC is described in [1]. Video being a 3 dimensional signal with 2 dimensions in space and one in time is analysed using space time approach. The sequence of frames in a video is analysed according to the sequence of human motion in the activity and classification is done. This involves cameras for video capturing which make the system bulky and expensive. Another disadvantage of this method is the latency in processing and classification. The accuracy of such method may be improved using multiple cameras and better processing.

Optical motion capture system is one of the most accurate activity classification system in literature. This uses multiple cameras to capture the human motion from multiple angles and views. Thus a complete and precise idea of the activity is taken and then the classification is done. As a result, the method is exceptional in terms of its accuracy. However, such high accuracy may not be needed in many applications where cost effectiveness is a priority. The use of multiple cameras increases the cost very drastically and makes the system non-portable and bulky. As a result, a simple and cost-effective method is needed for most of the applications.

The next most commonly used method is based on the use of sensors like accelerometer, magnetometers, gyroscopes. However, the circuit may become useless if any one sensor fails to provide proper results. The optimized combination of the indications by all the sensors is needed and a combined interpretation needs to be drawn. This increases the computation complexity as the data from all the individual sensors is to be analysed. Conclusively, a system with simple design, low complexity and low cost is appreciated.

Thus, we arrive on the use of the signatures found in the reflection co-efficients of an on-body antenna to satisfy the given application in low cost and complexity. The reflection co-efficient of an antenna shows specific signatures with respect to distinct human activities when the antennas are mounted on body while activity performance. As a result if the signature is determined, we can classify the activity. Same principle is used in [2] where monopole antennas are used for HAC. The analysis has been done for both transmission as well as reflection co-efficient of on-body antennas while performing human daily activities. However, the size of antenna is very large in order to be employed on human body. Practically, monopole antennas can never prove to be optimum for on-body use for patients, human subjects. Thus, such system is not practical for any of the desired applications. The second drawback of the work presented in [2] is that it doesn't ensure the antenna to be body-mountable. Thus, SAR analysis is necessary before deployment of the antennas on human body. The system uses two antennas for S21 analysis and a single antenna for S11 analysis. 
[3] uses similar technique with only 1 on-body antenna and analysis of the signatures found in the reflection co-efficient. Here, exactly similar monopoles are used. The work reduces the bulkiness of the system than [2] but faces the same limitations of antenna size, portability and SAR measurements. The analysis of the data collected from the reflection co-efficient is done using DTW algorithm which gives computationally simple classification.

There are various other evidences in literature about this method but the huge size of antenna remains a constant barrier. [3-5] specify such methods and their results for HAC. The above discussion gives a strong motivation for the design of miniaturized on body antennas and performance of efficient HAC using these antennas.

We started with the thought of removing the barrier of wired antennas as this stood as the biggest problem in portability. Thus, we designed a patch antenna which could be used for HAC. The most optimum frequency for the same was thought to be $915 \mathrm{MHz}$ as per the FCC regulations. A patch antenna was designed successfully and the entire HAC exercise was performed.Further in order to reduce the size of antenna the most logical step was undertaken that is increasing the operating frequency. The frequency of $4.5 \mathrm{GHz}$ is approved by FCC or low power devices and hence an antenna was designed operating at this frequency. Various techniques were studied to get the most optimized way of antenna design. Patch antennas were thought to be the most suitable option for on body antenna system. Further, it has been stated in [6] that antennas drain less battery if Circularly polarized. The portability of the HAC being a main motive here, inspires to use circularly polarized (CP) antennas for optimum battery usage. A number of techniques are discussed in order to get $\mathrm{CP}$ to get an idea of performance of $\mathrm{CP}$ antennas with respect to their ARBW and resonances. It has to be noted that along with $\mathrm{CP}$, the antenna also needs to be body-deployable.

The present work thus focuses on antenna miniaturization and HAC using these antennas. The first milestone is the shift from monopole antenna to a patch antenna. HAC is performed using a microstrip patch antenna. Keeping the aspect of $\mathrm{CP}$ in mind, another antenna is designed which is further miniaturized and equipped with $\mathrm{CP}$. The $\mathrm{CP}$ is obtained by using a innovative complimentary ground technique. Finally, the analysis of the data collected from the reflection co-efficients of these antennas is done using DTW algorithm.

\section{Antenna Design Methodology}

Having studied different methods of antenna design from literature, Two antennas have been designed, fabricated and tested in the present work. The results of both the antennas are then compared. The first antenna is the conversion of the dipole antenna as used in [2] into a simple patch antenna. The antenna operates at $915 \mathrm{MHz}$ which is the most common frequency used for WBAN communication. The antenna consists of a simple rectangular patch with dimensions as shown in the Fig. 1. It is much more compact as compared to the antennas used for HAC in literature.

In order to make the antenna even more compact and address the issue of battery consumption; another compact, $\mathrm{CP}$ microstrip antenna has been designed for HAC. The antenna is designed using the innovative concept of complementary ground plane for obtaining CP. The antenna works at $4.5 \mathrm{GHz}$ which falls in high frequency WBAN band. 
Fig. 1 Patch antenna for HAC resonating at $915 \mathrm{MHz}$ (Antenna A)

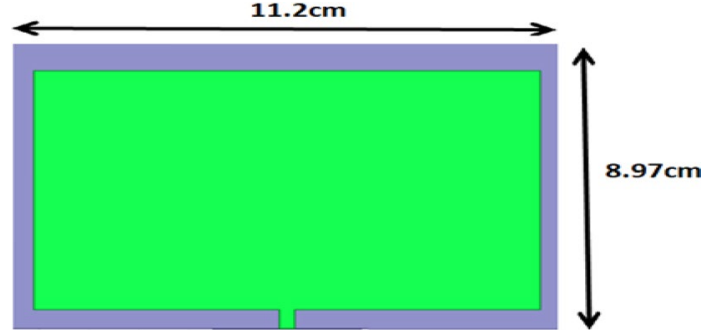

W
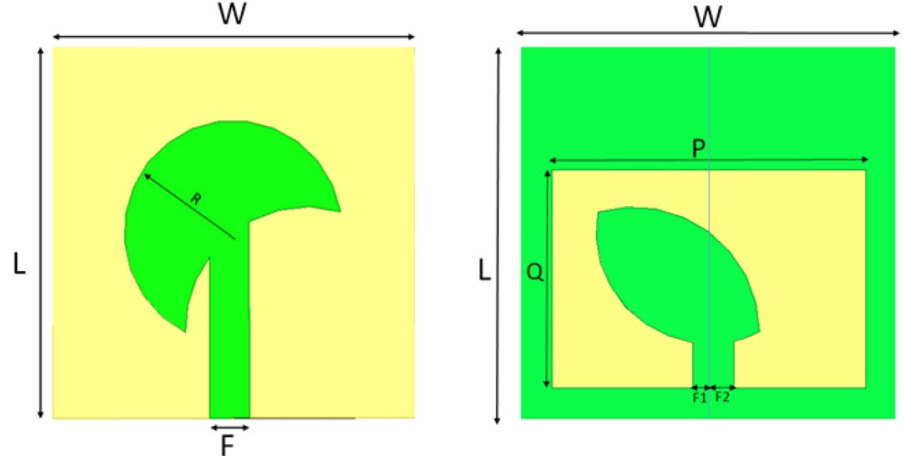

Fig. 2 Patch antenna for $\mathrm{HAC}$ resonating at $4.5 \mathrm{GHz}$ (Antenna B)

The up-shift in the working frequency leads to miniaturization of the antenna. The antenna design and configuration can be seen in Fig. 2.

The dimensions of antenna $\mathrm{B}$ are as follows: $\mathrm{R}=10 \mathrm{~mm}, \mathrm{~F}=3.3 \mathrm{~mm}, \mathrm{~F} 1=1.3 \mathrm{~mm}$, $\mathrm{F} 2=2 \mathrm{~mm}, \mathrm{~L}=30 \mathrm{~mm}, \mathrm{~W}=30 \mathrm{~mm}, \mathrm{P}=25 \mathrm{~mm}, \mathrm{Q}=17.5 \mathrm{~mm}$ It can be seen from Fig. 2 that the ground plain exactly compliments the patch. Further, the frame is added in the ground plane for proper impedance matching.

The two compact antennas designed were simulated with human body phantom and SAR measurements were taken inorder to confirm if the antennas can be mounted on human body.

\section{Antenna Simulation with Human Phantom}

The present application makes in use of antennas as a part of HAC in the following wayThe antennas to be employed for HAC will be mounted on human wrist. Further the human subject with antenna on wrist will perform the activity. The dataset of the reflection co-efficient of antenna will be thus collected in real time and will be compared with the existing datasets for pre-defined activities which the classifier ought to classify. Thus while simulating the antenna, we need to simulate it with human wrist phantom. The human body phantom was adopted from [7]. Three layers on human body -skin, fats and muscle were considered (Fig. 3) and simulated in HFSS along with the designed antennas. The human wrist phantom model with antenna can be seen in Fig. 4. The values of di-electric constants for skin, fat and muscle are frequency dependent and thus are set as per the operating 


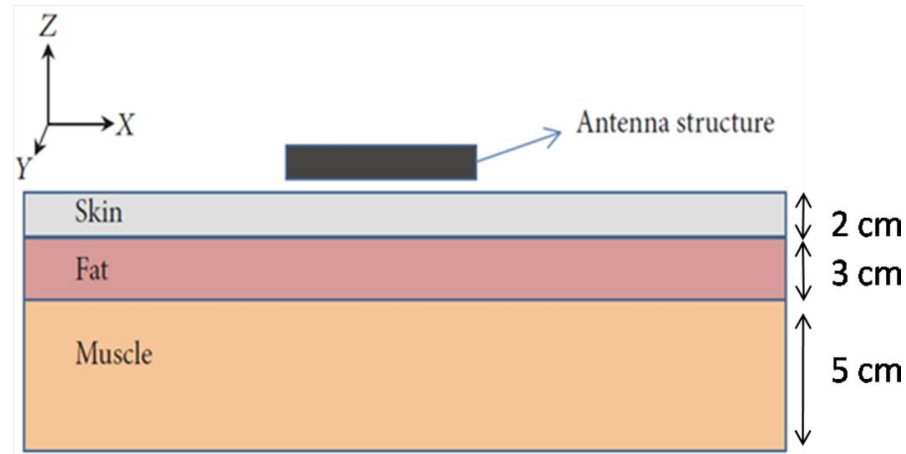

Fig. 3 Human wrist phantom

Fig. 4 Human wrist phantom with Antenna A

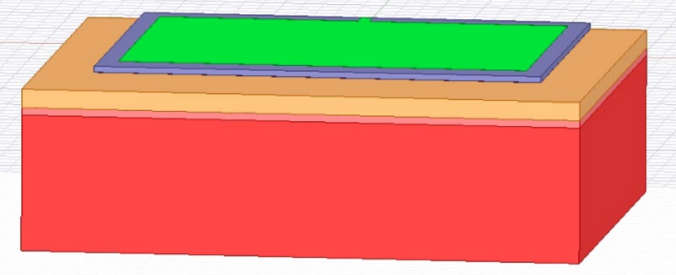

frequency of the antenna as mentioned in [8]. The simulations for Antenna A are discussed which applies similarly to Antenna B .

\section{Dataset Collection}

A very intuitive procedure was followed for dataset collection where the reflection coefficient of the antenna was measured at different position indices while activity performance. The reference dataset was formed by performing same activity with different subjects multiple times (Reference signals generated when plotted against position indices). To get a thorough diversity of subjects, 5 male and 5 female subjects were selected for activity performance. Their weight varied from $40 \mathrm{Kg}$ to $70 \mathrm{Kg}$ while height was in range 150-180 $\mathrm{cm}$. The antenna was kept in close proximity of the wrist for each subject and they were told to perform the mentioned rhythmic actions multiple times. The experiment was performed in different times of the day and in different surroundings to get an idea of change in trend with respect to surroundings. It was observed that the variation in trend with respect to environment was minimal as the fields being explored are antenna near fields which are in range of cms. When a test activity is performed, the euclidean distance of this test signal was to be found with respect to the reference signals of all the considered activities using DTW algorithm and the activity 
Table 1 SAR Measurement values

Table 2 Eu distance values for three different actions for Antenna A

\begin{tabular}{llll}
\hline Antenna & Skin & fat & Muscle \\
\hline A & $0.11 \mathrm{~W} / \mathrm{Kg}$ & $0.875 \mathrm{~W} / \mathrm{Kg}$ & $0.019 \mathrm{~W} / \mathrm{Kg}$ \\
B & $0.25 \mathrm{~W} / \mathrm{Kg}$ & $0.751 \mathrm{~W} / \mathrm{Kg}$ & $0.025 \mathrm{~W} / \mathrm{Kg}$ \\
\hline
\end{tabular}

\begin{tabular}{lccc}
\hline AntennaA & WAVING & BOXING & CLAPPING \\
\hline WAVING & 2.68 & 15.49 & 38.65 \\
BOXING & 10.99 & 4.20 & 37.75 \\
CLAPPING & 39.06 & 38.08 & 0.00 \\
\hline
\end{tabular}

Table 3 Eu distance values for three different actions for Antenna B

\begin{tabular}{lccc}
\hline AntennaB & WAVING & BOXING & CLAPPING \\
\hline WAVING & 74.88 & 114.94 & 324.79 \\
BOXING & 153.14 & 25.89 & 435.08 \\
CLAPPING & 497.72 & 550.49 & 0.00 \\
\hline
\end{tabular}

with minimum euclidean distance should be selected. Same process was followed in [3]. However, the number of samples considered in present work was drastically reduced as compared to [3] due to change in the measurement method. Vector Network AnalyserVNA N99233A was used to collected the dataset in the present work.

\section{Experimental Results}

The antennas quoted in the above sections were fabricated and tested. The measured return loss performance of the antennas is in very good agreement with the simulations (Fig. 5, 6). Same holds for the axial ratio performance (Fig. 7). The Co and Cross Polarized radiation pattern of proposed antennas in $\mathrm{E}$ and $\mathrm{H}$ plane can be observed in Figs. 8 and 9. The dataset was then collected using the fabricated antennas (Fig. 10 and 11).

The fabricated antennas were further employed for HAC set-up. The SAR measurements with the human wrist phantom proved both the antennas to be suitable for mounting on human body. Table. 1 depicts the SAR values in three considered layers of human body for both the antennas. The general limit over SAR value in body is $2 \mathrm{~W} / \mathrm{Kg}$ and thus as per the measurements, the antennas are optimum for on body use as the SAR is below $2 \mathrm{~W} / \mathrm{Kg}$ in all the layers of considered phantom. The antennas were tied on the wrist of the subject performing the activity. Precautions were taken in order to keep the antenna in same position throughout the activity performance.

The return loss of the antenna was recorded at 9 different points while activity performance. The return loss was then plotted against these 9 position indices. This signal which was plotted (S11 vs position index) showed same signatures for same activity irrespective to the time, place at which the activity was performed and subject who performs the activity. Fig. 12, 13, 14 depict the S11 signatures for Antenna A for three activities- Waving, 


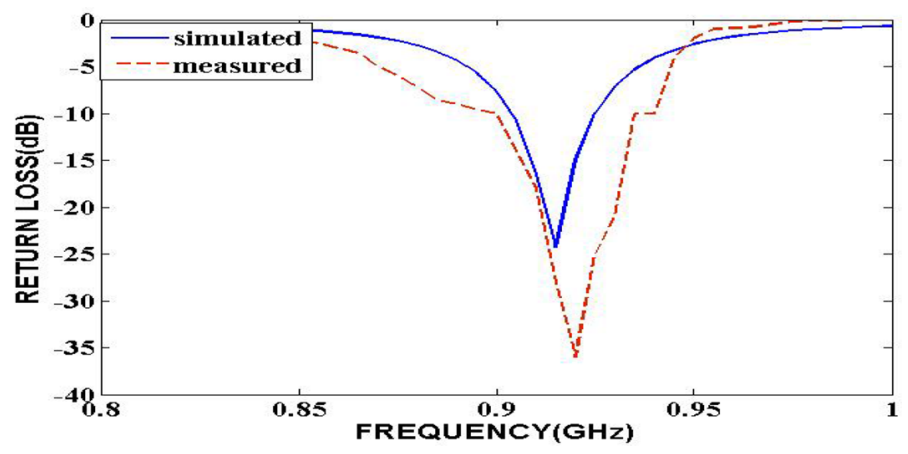

Fig. 5 Return Loss performance of Antenna A

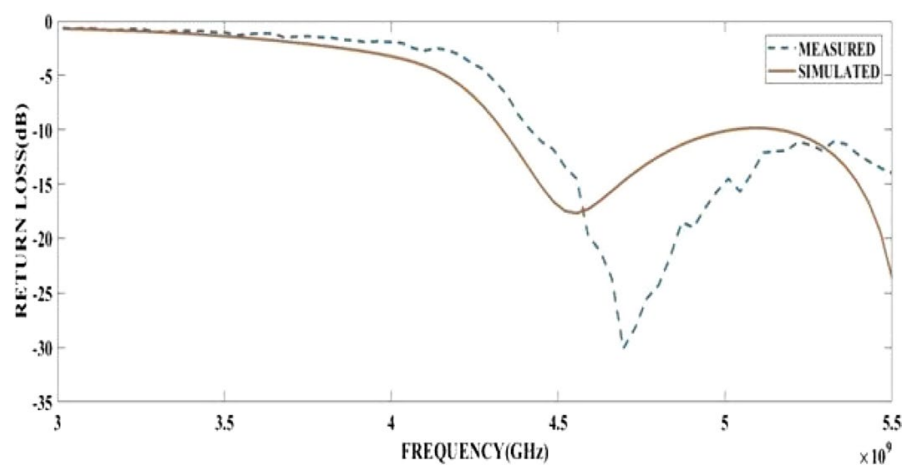

Fig. 6 Return loss performance of Antenna B

Fig. 7 Axial ratio performance for Antenna B

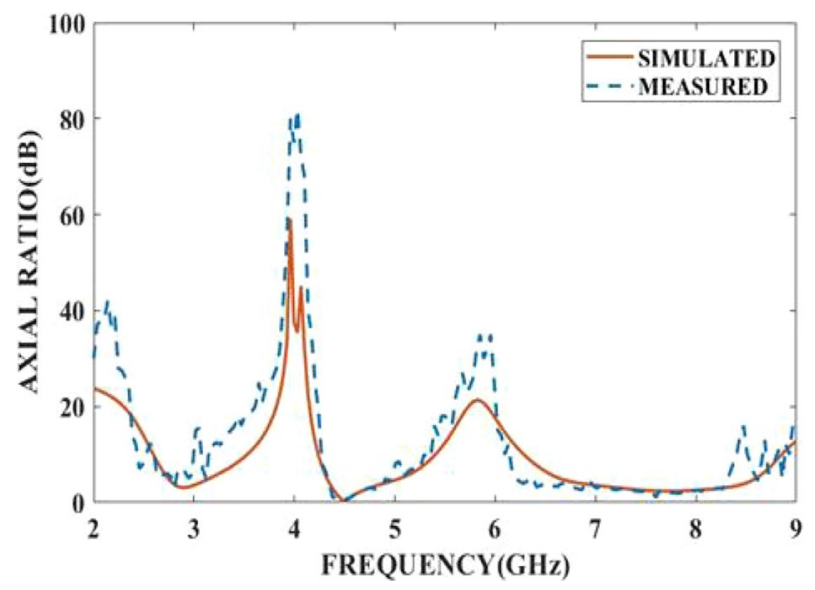




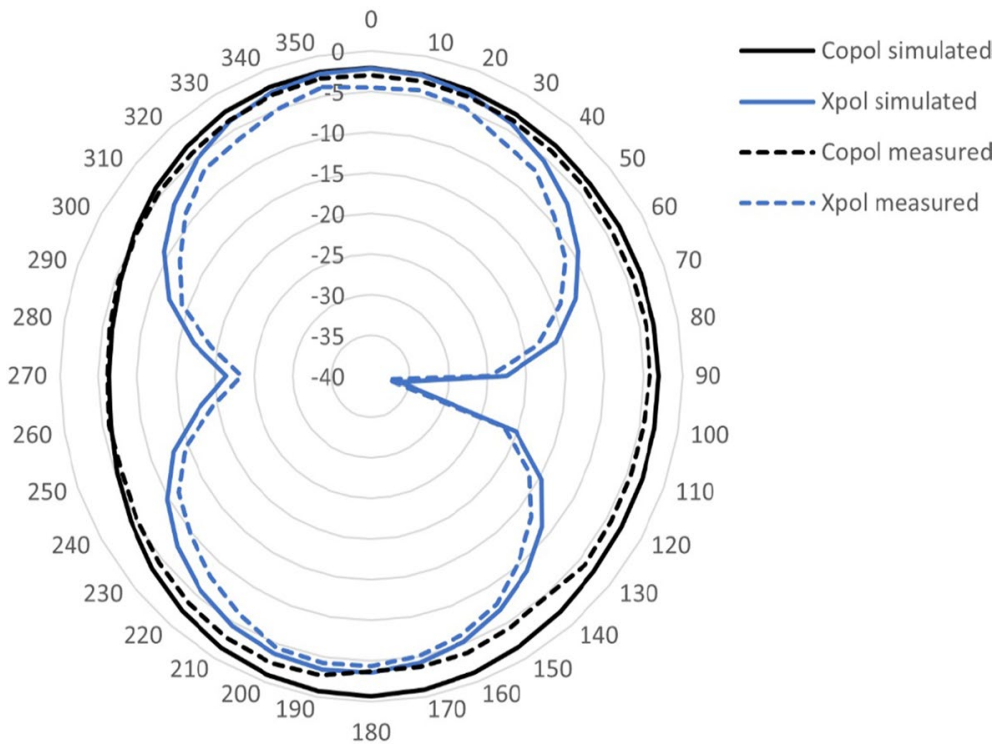

Fig. 8 Simulated \& Measured Radiation Pattern in E plane for Antenna B

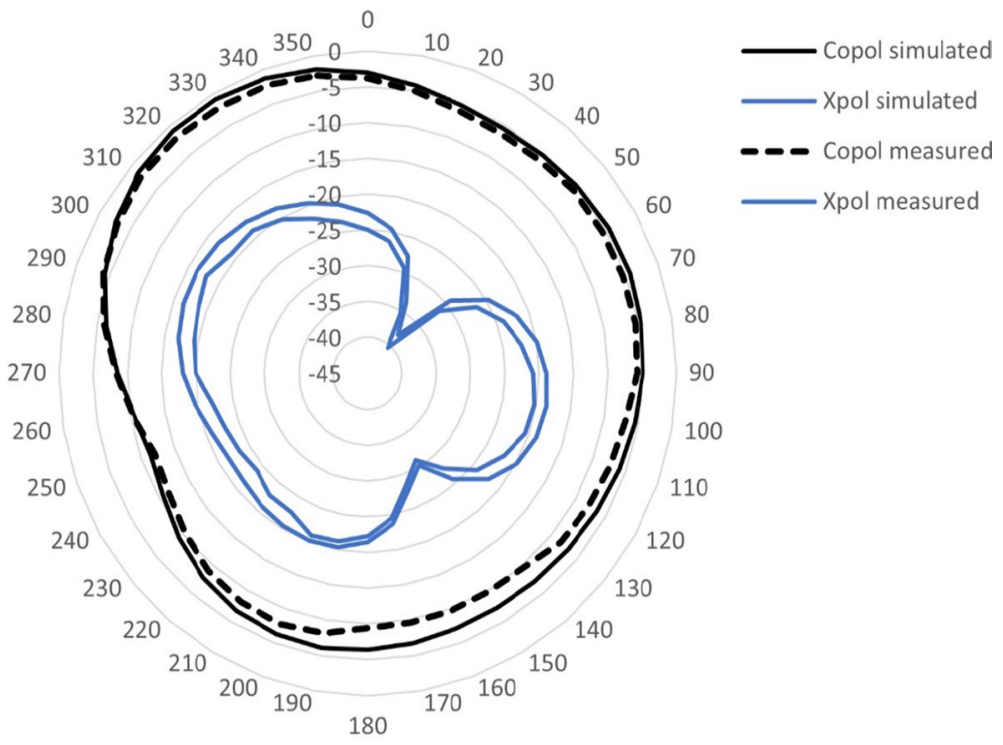

Fig. 9 Simulated \& measured radiation pattern in $\mathrm{H}$ plane for Antenna B

Clapping and boxing while the signatures for Antenna B can be seen in Figs. 15, 16, 17 for same three activities.

It can be concluded visually that specific trends/signatures are obtained for different activities irrespective of the subject who performs it. However, in order to analyse the situation analytically, DTW algorithm was used. The inter-euclidean(Eu) distance between 
Fig. 10 Image of fabricated Antenna A
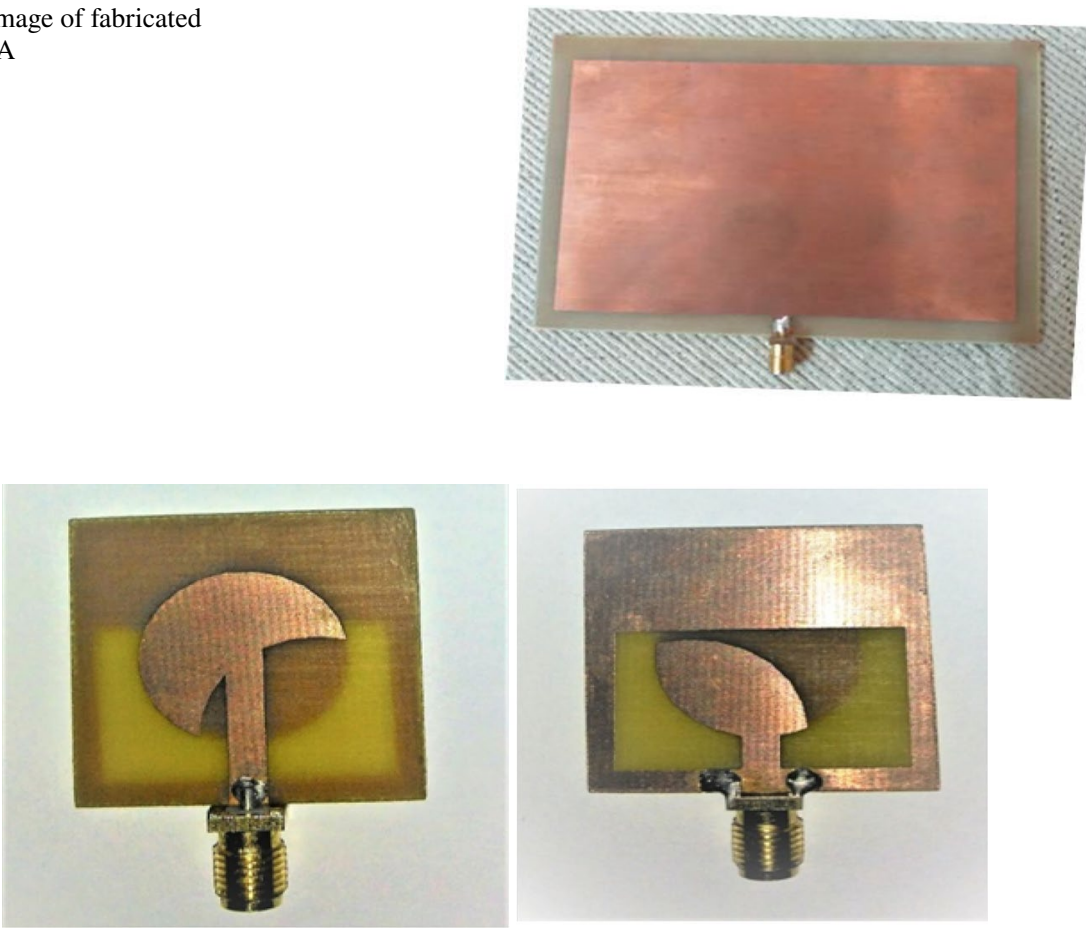

Fig. 11 Image of fabricated Antenna B

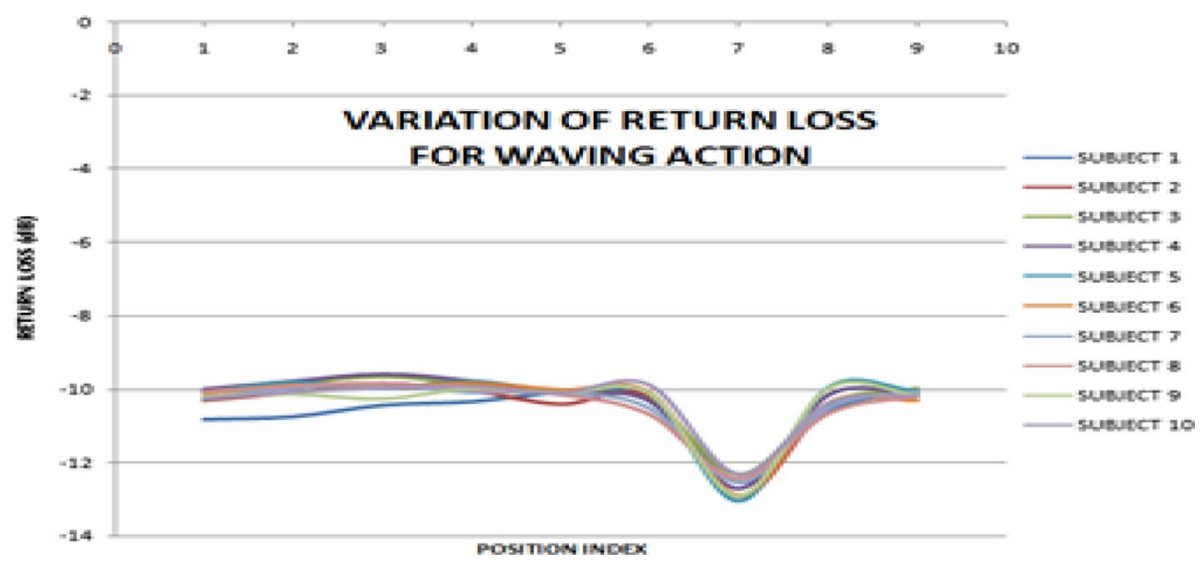

Fig. 12 Signatures of reflection co-efficient for waving action using Antenna A

each signal for an activity was found with the reference signals of other activities and the average (of Eu distance) was taken. The resultant value tells on an average how far the signal obtained from one activity is from the other activity. The Table. 2 and Table 3 show the resultant distance values. It can be seen that the Eu distance between the signals obtained from the same activity is very low as compared to the Eu distance between 


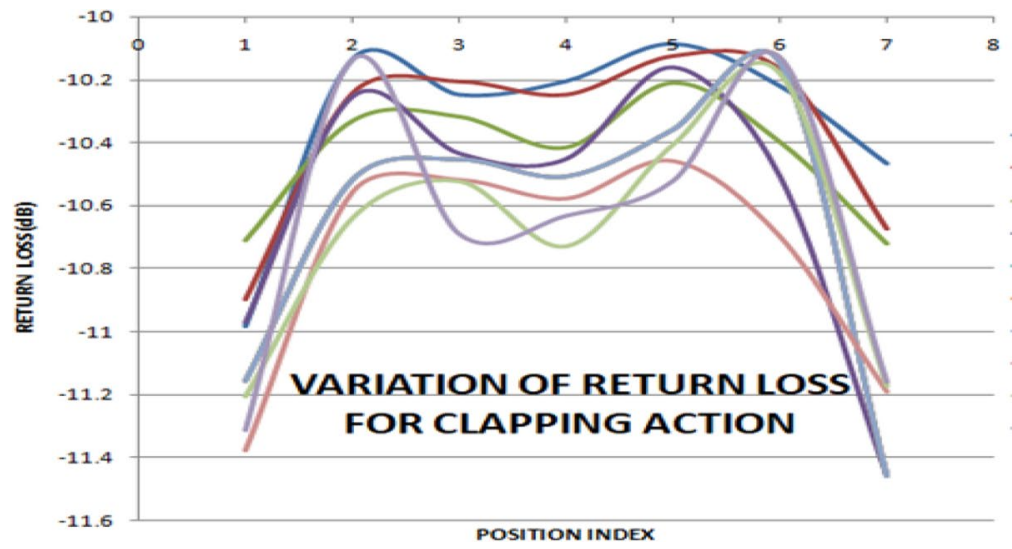

SUBJECT 1

SUBJECT 2

SUBUECT 3

SUBUECT 4

SUBUECT 5

SUBUECT 6

SUBJECT 7

SUBJECT 8

SUBJECT 9

SUBJECT 10

Fig. 13 Signatures of reflection co-efficient for clapping action using Antenna A

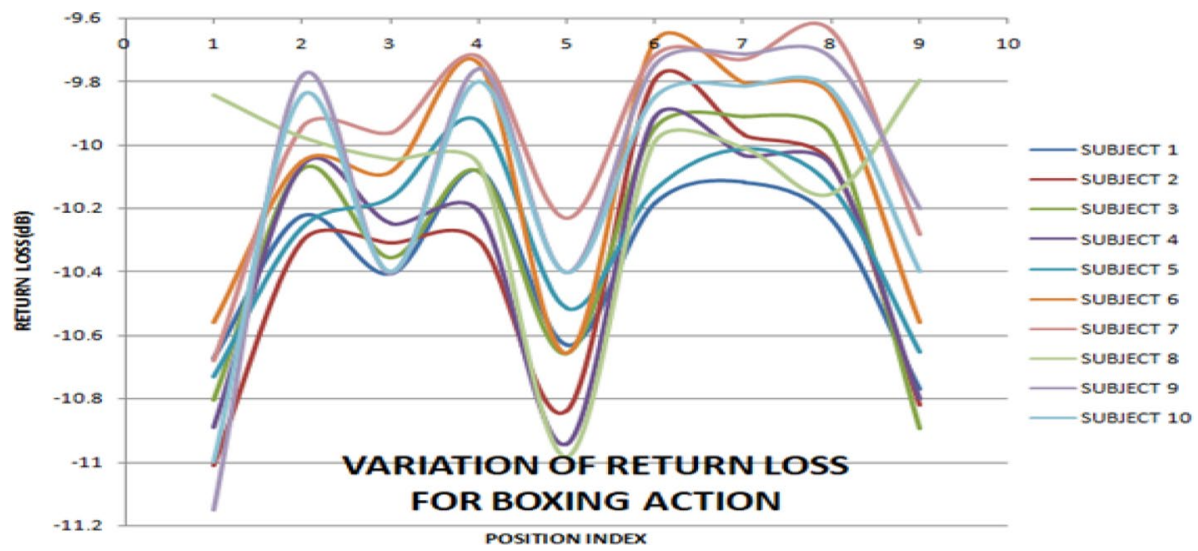

Fig. 14 Signatures of reflection co-efficient for boxing action using Antenna A
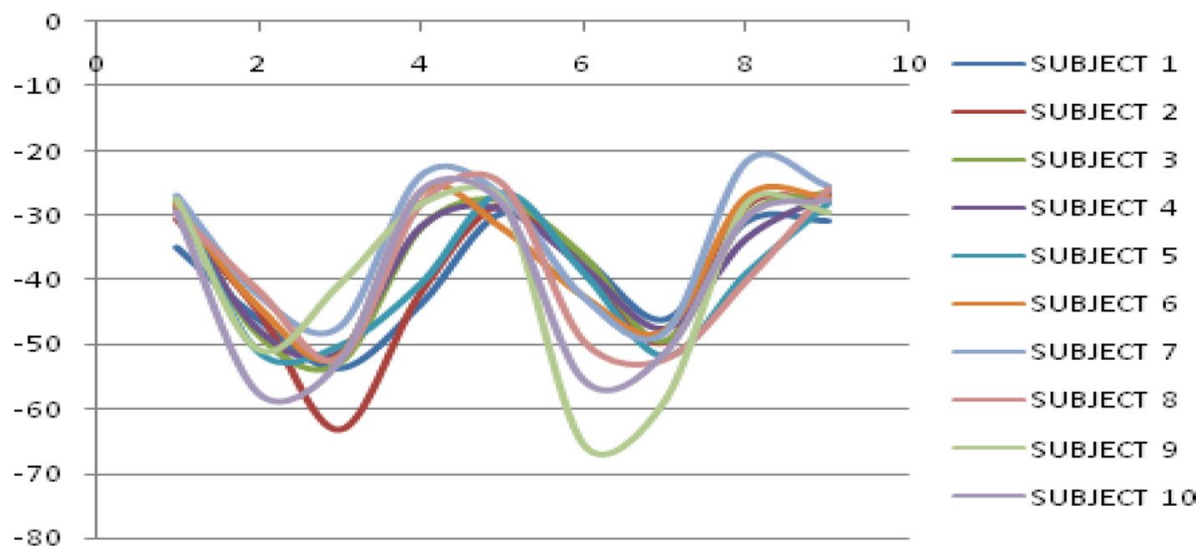

Fig. 15 Signatures of reflection co-efficient for waving action using Antenna B 


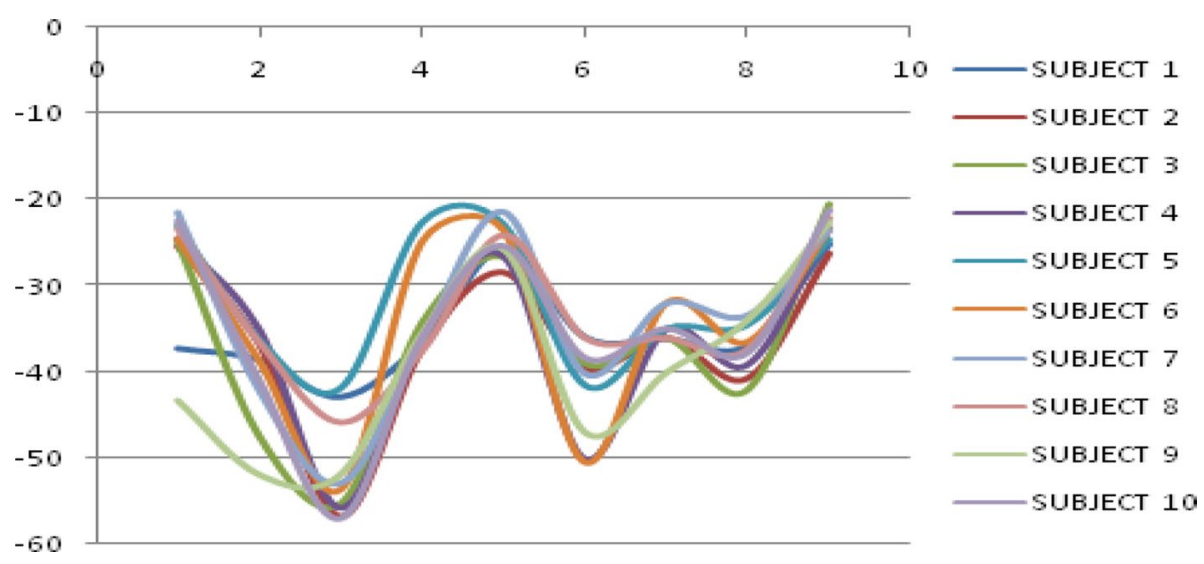

Fig. 16 Signatures of reflection co-efficient for clapping action using Antenna B
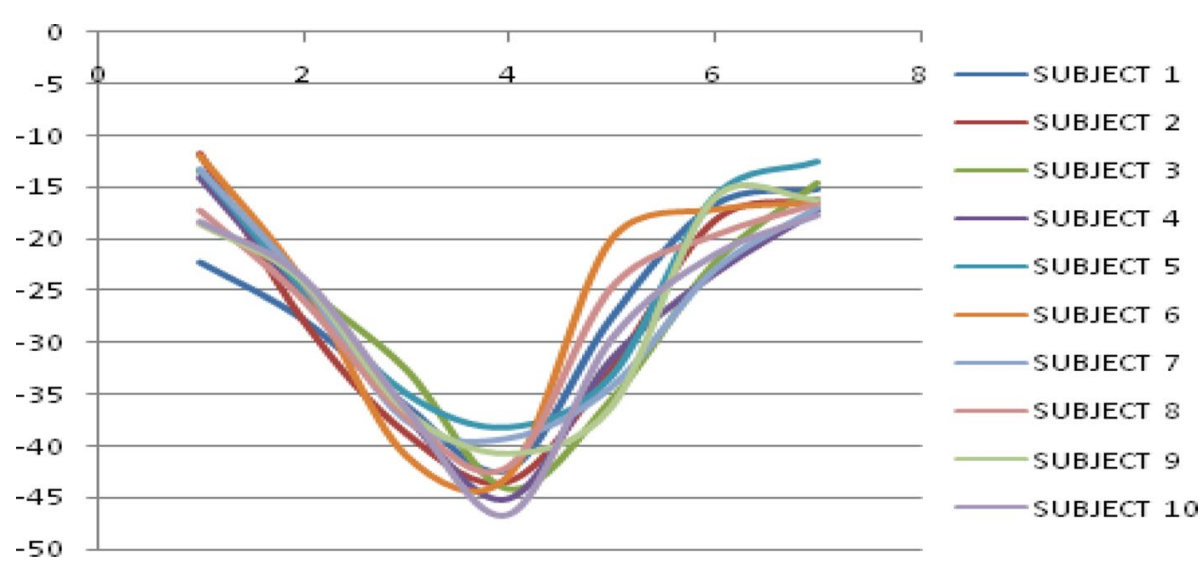

Fig. 17 Signatures of reflection co-efficient for boxing action using Antenna B

signals of different activities. Thus we prove analytically that specific signatures are successfully obtained in the reflection co-efficients of both the antennas for the considered activities. Also, the signatures of two different activities vary drastically which allows to easily finalize a decision boundary for classification. It is worth to note that Antenna B performs better in terms of inter-activity Eu. distance and making a decision would be simpler for antenna B as the difference of Eu distance is maximum. Thus, from large monopoles quoted in literature with no SAR optimization, we arrived on miniaturized $(3 \mathrm{~cm} \times 3 \mathrm{~cm})$ compact patch antennas satisfying exactly the same purpose with controlled SAR. It can be concluded that simple, cost-effective, compact and practically implementable $\mathrm{HACl}$ has been designed successfully. 
Table 4 Characteristics of some CP Antennas in Literature

\begin{tabular}{lllllll}
\hline Ref & $\begin{array}{l}\text { Resonant } \\
\text { frequency }\end{array}$ & Size & Return loss & Axial ratio & BW & ARBW \\
\hline$[9]$ & $10.13 \mathrm{GHz}$ & - & $-45 \mathrm{~dB}$ & $1.286 \mathrm{~dB}$ & $500 \mathrm{MHz}$ & $80 \mathrm{MHz}$ \\
{$[10]$} & $2.45 \mathrm{GHz}$ & $0.491 \lambda * 0.491 \lambda$ & $-45 \mathrm{~dB}$ & $1.1 \mathrm{~dB}$ & $182 \mathrm{MHz}$ & $40 \mathrm{MHz}$ \\
{$[11]$} & $5.75 \mathrm{GHz}$ & $0.86 \lambda * 0.86 \lambda$ & $-30 \mathrm{~dB}$ & $1.9 \mathrm{~dB}$ & $1.75 \mathrm{GHz}$ & $900 \mathrm{MHz}$ \\
{$[12]$} & $2.7 \mathrm{GHz}$ & $0.72 \lambda * 0.72 \lambda$ & $-20 \mathrm{~dB}$ & $3 \mathrm{~dB}$ & $1.02 \mathrm{GHz}$ & $590 \mathrm{MHz}$ \\
{$[13]$} & $1.61 \mathrm{GHz}$ & $0.307 \lambda * 0.307 \lambda$ & $-35 \mathrm{~dB}$ & $3 \mathrm{~dB}$ & $60 \mathrm{MHz}$ & $45 \mathrm{MHz}$ \\
$\begin{array}{c}\text { Proposed } \\
\text { Antenna }\end{array}$ & $4.55 \mathrm{GHz}$ & $0.455 \lambda * 0.455 \lambda$ & $-17.86 \mathrm{~dB}$ & $1.1 \mathrm{~dB}$ & $1.5 \mathrm{GHz}$ & $380 \mathrm{MHz}$ \\
\hline
\end{tabular}

\section{Discussion}

The work primarily focuses on the use of antenna and its near fields combined as sensors to detect motion, human activities in specific. A very versatile feature of antenna theory has been exploited that the near fields of antenna are sensitive to perturbations and hence show very specific trend with respect to distinct actions a human might perform. As the entire system relies on antenna design and performance, very optimum antenna was designed which possess all characteristics required for HAC and it was found to outperform when bench-marked with other antennas available in literature (Table 4). It was found that this single concept can be applied in numerous other fields like rehabilitation and as gesture recognizer for handicapped. The current work is also being extended to classification of COVID-19 specific actions like coughing, sneezing and touching the face. Classification of such actions can surely help in reducing the spread of corona virus. Thus the paper discusses the end to end specifications of the HAC using miniature antennas which states that the desired goal has been achieved.

\section{Conclusion}

A compact and cost effective HAC1 has been proposed in the paper. The huge, practically non-implementable dipole antennas for this application in literature can now be replaced with small patch antennas. The antenna B being circularly polarized would also help in battery life management when the system is deployed in battery operated devices in practice. The antennas are suitable to be mounted on body as proved by the SAR analysis. The antennas were tied on human wrist and actual data set was collected. The collected data was analysed using DTW algorithm and the Eu. distance results proved that that antennas reflection co-efficient is showing specific signatures for the considered human daily activities. The work can be further improvised by collecting more and more data and increasing the number of activities to be classified.

Funding This work was not funded by any agency.

Data Availibility The datasets generated during and/or analysed during the current study are available from the corresponding author on reasonable request. 
Code Availibility The code used (if any) in the manuscript is a generalized code for data analysis and is not specific to the findings of this work.

\section{Declarations}

Conflicts of interest There is no conflict of interest in publishing this manuscript.

Competing interests There is no conflict of interest in publishing this manuscript.

Data Transparency The data obtained in this work is clearly presented in the manuscript and available with the author.

\section{References}

1. Aggarwal, J. K., \& Ryoo, M. S. (2011). Human activity analysis: A review. ACM Computing Surveys (CSUR), 43(3), 16.

2. Kim, Y., \& Li, Y. (2017). Human activity classification with transmission and reflection coefficients of on-body antennas through deep convolutional neural networks. IEEE Transactions on Antennas and Propagation, 65(5), 2764-2768.

3. Li, Y., \& Kim, Y. (2016). Classification of human activities using variation in impedance of single on-body antenna. IEEE Antennas and Wireless Propagation Letters, 16, 541-544.

4. Bresnahan, D., Li, Y., Koziol, S., \& Kim, Y. (2018). Monitoring human head and neck-based motions from around-neck creeping wave propagations. IEEE Antennas and Wireless Propagation Letters, 17(7), 1199-1203.

5. Alnujaim, I., Alali, H., Khan, F., \& Kim, Y. (2018). Hand gesture recognition using input impedance variation of two antennas with transfer learning. IEEE Sensors Journal, 18(10), 4129-4135.

6. Kaivanto, E., Salonen, E., \& Khaleel, H. (2014). Circularly polarized wearable antennas. WIT Transactions on State-of-the-Art in Science and Engineering, 82, 145-161.

7. Doddipalli, S., Kothari, A. and Peshwe, P., (2017). A low profile ultrawide band monopole antenna for wearable applications. International Journal of Antennas and Propagation.

8. Çelik, D., (2013). Computation of the cell phone-induced SAR distribution in a 3 D multi-layered model of the human head. Brain Using ANSYS.

9. Rahman, M.A., Hossain, Q.D., Hossain, M.A. and Chowdhury, P., (2014), May. Design of a circular polarization array antenna with dual-orthogonal feed circuit. In: 2014 International conference on informatics, electronics \& vision (ICIEV) (pp. 1-5). IEEE.

10. Liao, C.T., Lin, Y.F., Chen, C.H., Kao, S.Y. and Chen, H.M., (2016). A novel aperture-coupled circularly polarized square-ring patch antenna for wireless communication systems. In: 2016 IEEE 5th asia-pacific conference on antennas and propagation (APCAP) (pp. 57-58). IEEE.

11. Maddio, S. (2015). A compact wideband circularly polarized antenna array for $C$-band applications. IEEE Antennas and Wireless Propagation Letters, 14, 1081-1084.

12. Cai, Y.M., Li, K. and Yin, Y., (2017). A wideband circularly polarized microstrip slot-patch antenna design. In: 2017 IEEE Asia pacific microwave conference (APMC) (pp. 1185-1187). IEEE.

13. Rao, K.S., Jahagirdar, D.R. and Ramakrishna, D., (2017). Compact broadband asymmetric slit Circularly Polarized Microstrip Patch Antenna for GPS and GLONASS applications. In: 2017 IEEE International conference on antenna innovations \& modern technologies for ground, aircraft and satellite applications (iAIM) (pp. 1-3). IEEE. 
Publisher's Note Springer Nature remains neutral with regard to jurisdictional claims in published maps and institutional affiliations.
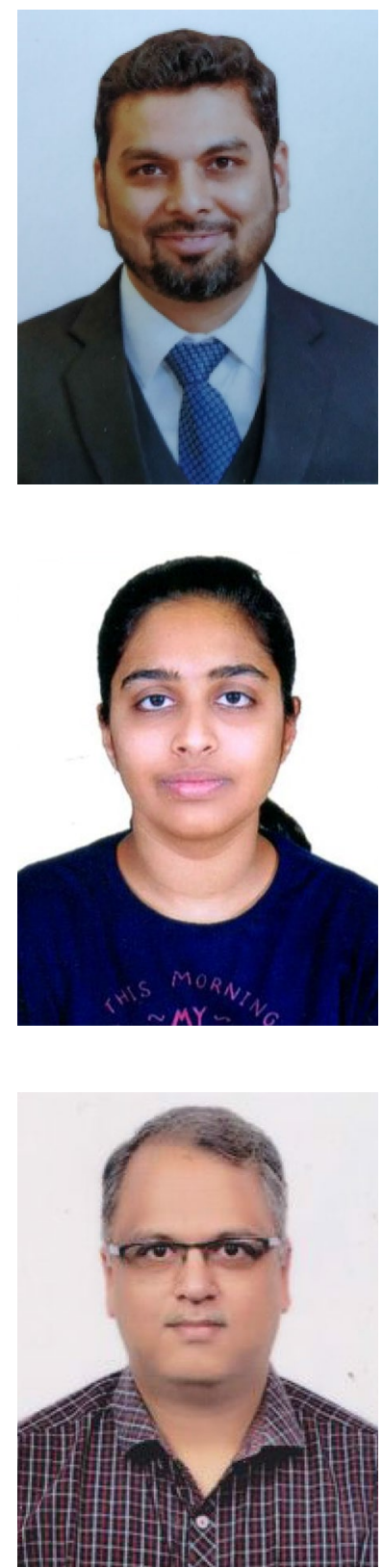

Paritosh D. Peshwe received B.E. degree in Electronics and Telecommunication Engineering in 2010. He further obtained M.E. degree in Microelectronics from Birla Institute of Technology \& Science, Pilani-Goa, India in 2013. He received Ph.D. degree from Visvesvaraya National Institute of Technology, Nagpur, India in 2019. He is currently working as an assistant professor in Indian Institute of Information Technology, Nagpur. His research areas include antenna design, metamaterials and wireless communication.

Neha Y. Joshi received her B.tech from Visvesvaraya National Institute of Technology, Nagpur. Her research areas include antenna design and IoT.

Ashwin Kothari received B.E. and M. Tech. degrees in Electronics Engineering in 1994 and 2005. He obtained Ph.D. degree from Visvesvaraya National Institute of Technology, Nagpur, India in 2010, where he is currently working as an Associate Professor. He is one of the coordinators for Center of Excellence of COMMBEDDED SYSTEMS: Hybridization of Communications and Embedded Systems under World Bank assisted project of TEQIP 1.2.1. He has authored around 25 publications and has contributed book chapters for reputed publications. He has 20 years of experience in teaching. His research areas include antennas and wave propagation, electromagnetics, communication and Rough Sets. 